\title{
Suppression of CEP55 reduces cell viability and induces apoptosis in human lung cancer
}

\author{
LIGANG LIU $^{2}$, QI MEI ${ }^{1}$, JING ZHAO ${ }^{1}$, YUHONG DAI ${ }^{1}$ and QIANG FU $^{1}$ \\ Departments of ${ }^{1}$ Oncology and ${ }^{2}$ Cardiothoracic Surgery, Tongji Hospital, Tongji Medical College, \\ Huazhong University of Science and Technology, Wuhan, Hubei 430030, P.R. China
}

Received April 6, 2016; Accepted May 12, 2016

DOI: 10.3892/or.2016.5059

\begin{abstract}
Centrosomal protein 55 (CEP55), identified as a centrosome-associated protein, has been reported to be involved in human malignancies. However, its biological function in human lung cancer remains largely unknown. In the present study, we firstly analyzed the expression of CEP55 in 20 pairs of lung cancer and matched non-tumor tissues using quantitative RT-PCR analysis and found that CEP55 mRNA was significantly increased in lung cancer tissues compared with that in matched tumor-adjacent tissues. Then we performed a loss-of-function assay using lung cancer cell lines A549 and 95D. Functionally, knockdown of CEP55 markedly inhibited cell viability and proliferation ability as determined by MTT and colony formation assays. Moreover, CEP55-silenced cells were obviously arrested in the G0/G1 phase and presented significant cell apoptosis as determined using flow cytometric analysis. Mechanistically, western blot analysis further revealed that knockdown of CEP55 decreased the expression of CDK4, p21 and Bcl-2, while it increased the expression of pro-apoptotic protein, Bad, caspase-3 and PARP in $95 \mathrm{D}$ cells. In conclusion, our data highlight the crucial role of CEP55 in promoting lung cancer cell proliferation in vitro and its inhibition may be a novel therapeutic strategy for lung cancer.
\end{abstract}

\section{Introduction}

Lung cancer, mostly caused by smoking and exposure to pollutants, is one of the leading causes of death worldwide (1). According to its histological types, lung cancer is classified into small cell lung cancer and non-small cell lung cancer (NSCLC). For many years, the existing therapeutic

Correspondence to: Dr Qiang Fu, Department of Oncology, Tongji Hospital, Tongji Medical College, Huazhong University of Science and Technology, 1095 Jie fang Avenue, Hankou, Wuhan, Hubei 430030, P.R. China

E-mail: fqiang2016@163.com

Key words: centrosomal protein 55, lung cancer, cell viability, apoptosis, cell cycle strategies for lung cancer have been several traditional therapies, including surgery, chemotherapy and radiotherapy. Despite the advances in these traditional therapies, the mortality rates remain high, with an overall 5-year survival of only $15 \%$ (2). Therefore, comprehensive understanding of the molecular mechanisms underlying lung cancer progression may contribute to the effectiveness of anticancer therapy and thereby, the overall survival of lung cancer.

Centrosomal protein 55 (CEP55), also named C10orf3 (3) and URCC6, is the latest member found in the centrosomal relative protein family, which upon observation was revealed to localize to the centrosome in interphase cells, midzone during anaphase, and the midbody during cytokinesis by tagging with GFP-C (4-6). Furthermore, CEP55 has been identified as a microtubule-bundling protein and plays an important role in cell mitosis through cooperation with CDK1, ERK2 and PLK1 (4). Accumulated evidence has shown that CEP55 overexpression occurs in a wide range of solid tumors, including human colon (3), bladder cancer (7) and hepatocellular carcinoma tumorigenesis (8). In addition, CEP55 has been demonstrated to regulate critical cell functions including cell growth, transformation and cytokinesis. Overexpression of CEP55 was found to enhance cell cycle transition by activation of p21, whereas knockdown of CEP55 was found to inhibit cell growth in gastric (9) and breast cancer (10). Moreover, CEP55 was found to be upregulated by VEGFA in lung cancer tissues and associated with metastasis (11). In spite of these functional observations, the role of CEP55 in lung cancer has remained largely unclear.

The present study aimed to further investigate the biological function of CEP55 in lung cancer. We found that CEP55 was overexpressed in lung cancer tissues and cell lines. Our in vitro studies demonstrated that CEP55 promoted cell viability and proliferation through cell cycle progression and inhibition of apoptosis. These findings suggest that CEP55 overexpression is associated with tumor growth and may serve as a potential new therapeutic target for lung cancer.

\section{Materials and methods}

Clinical tissue specimens. A total of 20 fresh tumor tissue samples with paired non-cancerous lung tissue samples were derived from lung cancer patients who had undergone surgery at our institution. None of these patients had received radiotherapy 
or chemotherapy prior to surgical treatment. Dissected samples were frozen immediately after surgery and stored at $-80^{\circ} \mathrm{C}$ until use. All patients had signed written informed consents prior to the use of the clinical materials for this study.

Cell lines and culture. The human NSCLC cell lines H1299, A549, H128 and 95D were purchased from the Cell Bank of the Type Culture Collection of the Chinese Academy of Sciences (Shanghai, China). These cells were cultured in RPMI-1640 (Hyclone and Biowest) supplemented with 10\% fetal bovine serum (FBS) and incubated in a humidified atmosphere containing $5 \% \mathrm{CO}_{2}$. The medium was replaced every 2-3 days as indicated.

CEP55 short hairpin RNA (shRNA) stable transfection. Two CEP55 shRNA constructs, as well as a scrambled negative control (NC) shRNA were purchased from OriGene Technologies, Inc. (Rockville, MD, USA) and cloned into the pLKO.1-EGFP vector between AgeI and EcoRI restriction sites. Lentivirus particles were generated by co-transfecting recombined and packing vectors into $293 \mathrm{~T}$ cells via Lipofectamine 2000 (Invitrogen, Carlsbad, CA, USA). A549 and 95D cells were then cultured in 6-well plates and transfected with CEP55 shRNA-expression lentiviruses (sh-1 or -2) according to the manufacturer's instructions. All transfected cell lines were further evaluated for knockdown efficiency of the target gene (CEP55) using quantitative real-time PCR (qRT-PCR) and western blot analysis.

Quantitative RT-PCR. Total RNA was isolated using the TRIzol reagent (Invitrogen). Complementary DNA (cDNA) was synthesized from $2 \mu \mathrm{g}$ of total RNA using $200 \mathrm{U} / \mathrm{ml}$ SuperScript II Reverse Transcriptase (Invitrogen). Real-time PCR amplification reactions for CEP55 were carried out using the Bio-Rad Connect Real-Time PCR platform as previously reported (12). All reagents were purchased from Thermo Fisher Scientific (Waltham, MA, USA). The relative mRNA expression of CEP55 was normalized by using human GAPDH mRNA levels as an internal control. Data were analyzed using the $2^{-\Delta \Delta \mathrm{Ct}}$ method as previously described (13). Each experiment was performed in triplicate.

Protein isolation and western blot analysis. Total protein was isolated from cells using RIPA lysis buffer containing protease inhibitors (Sigma-Aldrich, St. Louis, MO, USA). The suspension was collected after centrifugation at $12,000 \mathrm{x}$ g for $15 \mathrm{~min}$ at $4{ }^{\circ} \mathrm{C}$, followed by protein concentration determination using BCA assay. Equal amounts of proteins (20-40 $\mu \mathrm{g})$ were separated on $10 \%$ sodium dodecyl sulfate-polyacrylamide gel (SDS-PAGE) and transferred to a PVDF membrane using a Bio-Rad semi-dry transfer system. Then the membrane was blocked with TBST (Tris-buffered saline, $0.1 \%$ Tween-20) containing 5\% non-fat dry milk for $1 \mathrm{~h}$ at room temperature, and probed with the corresponding primary antibodies overnight. Subsequently, the membrane was incubated with appropriated horseradish peroxidase-conjugated secondary antibodies (Santa Cruz Biotechnology, Inc., Santa Cruz, CA, USA) for $2 \mathrm{~h}$ at room temperature. Immunoreactive bands were visualized using Super ECL detection reagent (Applygen Technologies, Inc., Beijing, China).
Cell viability assay. MTT assay was used to determine cell viability in the A549 and 95D cells. Briefly, the cells were seeded into 96-well plates in triplicates at a density of 2,000 cells/well and cultured for $24 \mathrm{~h}$. Then $50 \mu \mathrm{l}$ MTT was added into each well at 1,2,3,4, and 5 days after transfection. After $4 \mathrm{~h}$ of incubation, $200 \mu 1$ dimethyl sulfoxide (DMSO) was added into each well. The absorbance value was measured using a microplate reader at $595 \mathrm{~nm}$. Each experiment was performed in triplicate.

Colony formation assay. To evaluate the effect of CEP55 on monolayer colony formation, stably transfected cells were seeded into 6-well plates at a density of 1,500 cells/well and allowed to grow for 7 days to form colonies. After being cultured for 7 days, the cells were fixed with methanol and stained with crystal violet. The number of colonies consisting of $>50$ cells/colony was counted under a microscope. Each experiment was performed in triplicate and repeated three times.

Cell cycle and apoptosis analysis. Cells were washed with cold PBS twice after a 48-h transfection and re-inoculated into $6-\mathrm{cm}$ dishes at a density of 80,000 cells/dish. Then the cells were collected and stained with propidium iodide (PI). The number of cells in each cell cycle phase was analyzed using a flow cytometer (FACSCalibur; BD Biosciences). In addition, apoptosis was determined by dual staining with an Annexin V-APC and PI apoptosis detection kit (Nanjing KeyGen Biotech, Co., Ltd., Nanjing, China) according to the manufacturer's instructions.

Statistical analysis. All statistical analyses were performed by SPSS software version 10.0 (SPSS, Inc., Chicago, IL, USA) and expressed as the mean \pm standard deviation (SD) of three independent experiments. Paired Student's t-test was used to compare differences between the groups. Statistically significant differences were accepted at $\mathrm{p}<0.05$.

\section{Results}

CEP55 is upregulated in lung cancer tissues and cell lines. To investigate the expression of CEP55 in lung cancer, we performed quantitative RT-PCR to detect CEP55 mRNA expression in 20 fresh tumor tissue samples with paired non-cancerous lung tissues. As shown in Fig. 1A, the mRNA level of CEP55 was significantly elevated in the lung cancer tissues compared with that noted in the adjacent normal tissues $(\mathrm{p}<0.001)$. Next, we further determined CEP55 expression in four lung cancer cell lines by western blot analysis. The results indicated that the protein level of CEP55 was obviously increased in all four lung cancer cell lines, among which it was expressed at a higher level in the A459 and 95D cells (Fig. 1B). Thus, A459 and 95D cells were chosen for the following studies.

The expression of CEP55 is significantly suppressed in lung cancer cells. To study the potential function of CEP55, we designed two shRNA constructs to specifically silence CEP55 expression in the A549 and 95D cells using a lentiviral system. Subsequently, we determined the knockdown efficiency on 
A

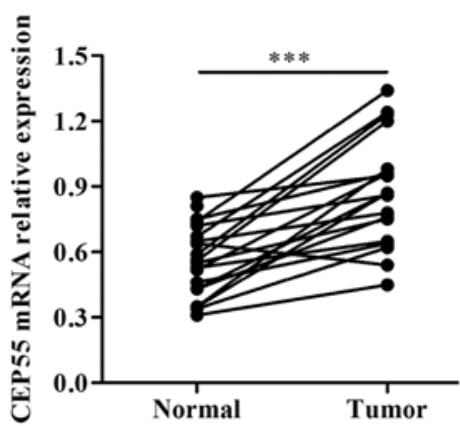

B

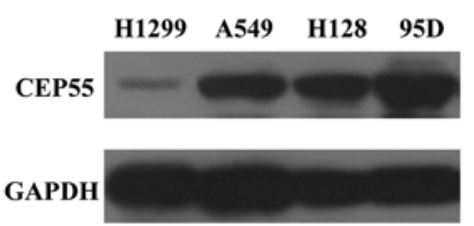

Figure 1. CEP55 is upregulated in lung cancer tumor tissues and cell lines. (A) CEP55 mRNA levels were determined by quantitative RT-PCR analysis in 20 fresh tumor tissue samples (Tumor) and paired non-cancerous lung tissue (Normal). (B) Western blot analysis of the CEP55 protein levels in four lung cancer cell lines. GAPDH was used as an internal control. ${ }^{* * *} \mathrm{P}<0.001$. CEP55, centrosomal protein 55.

A

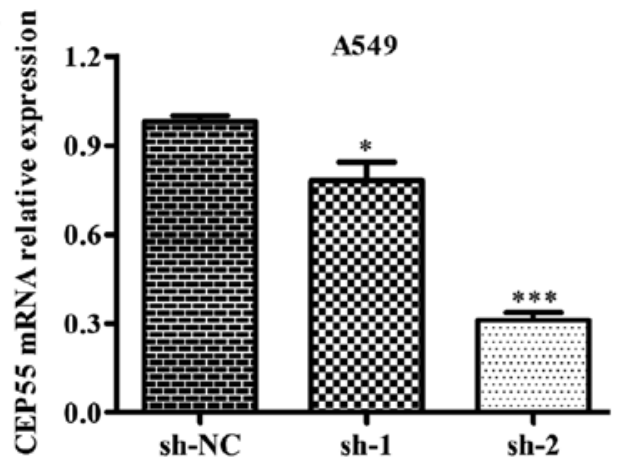

C

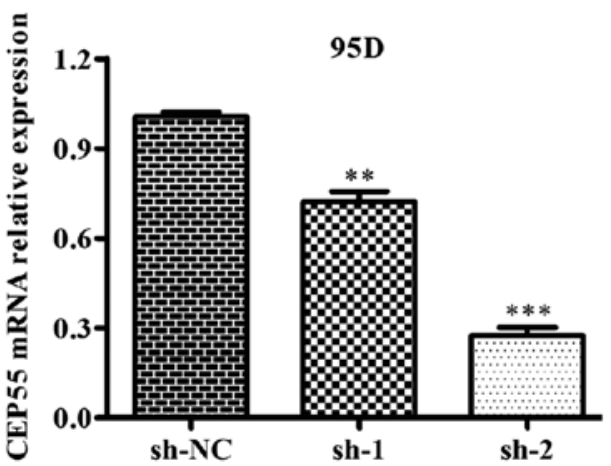

B

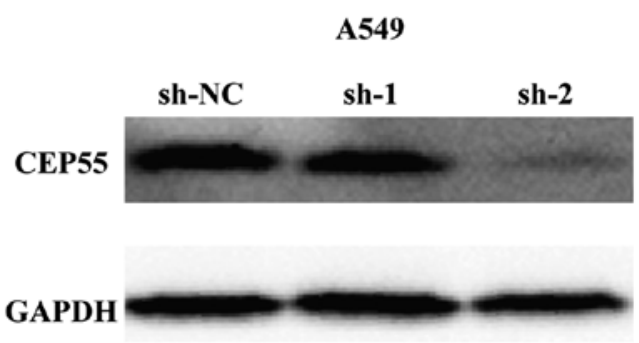

D

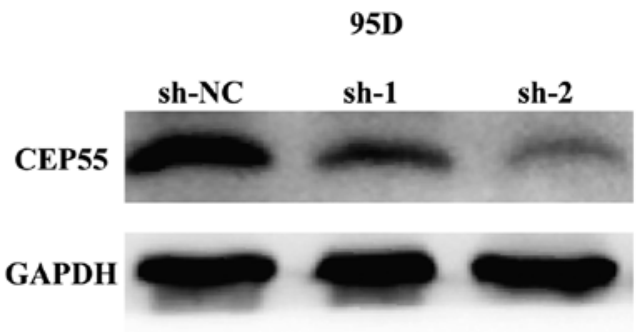

Figure 2. Expression of CEP55 was stably knocked down in the A549 and 95D cell lines. CEP55 mRNA levels were determined by quantitative RT-PCR in (A) the A549 and (C) 95D cells following transfection with a lentivirus containing two constructs of CEP55 shRNA (sh-1 and -2) or sh-NC. CEP55 protein levels were examined by western blot analysis in the (B) A549 and (D) 95D cells following transfection with the lentivirus containing two constructs of CEP55 shRNA (sh-1 and -2) or sh-NC. GAPDH was used as an internal control. $\mathrm{P}<0.05,{ }^{* *} \mathrm{p}<0.01,{ }^{* * * *} \mathrm{p}<0.001$. CEP55, centrosomal protein 55; shRNA, short hairpin RNA; NC, negative control.

CEP55 mRNA and protein levels in the A549 and 95D cells. As shown in Fig. 2A and C, the CEP55 mRNA level was significantly decreased in the sh-CEP55-infected cells. Notably, we found that the second construct $(\mathrm{sh}-2)(\mathrm{p}<0.001)$ showed better inhibiting efficiency than sh-1 $(\mathrm{p}<0.05, \mathrm{p}<0.01)$ in both cell lines compared with the first constructs (sh-1). Similarly, sh-2 suppressed the CEP55 protein level more markedly than sh-1 in the A549 and 95D cells (Fig. 2B and D). Therefore, sh-2 was selected for further experiments.

CEP55 knockdown inhibits lung cancer cell viability and colony formation ability. To investigate the inhibitory effect of CEP55 on cell growth, we measured cell viability using an MTT assay and found that the cell viability was significantly lower after five consecutive days in the CEP55-knockdown (sh-2) cells compared with that noted in the non-target scramble control shRNA-transfected cells (sh-NC) in both the A549 and 95D cell lines (Fig. 3A, p<0.001). Furthermore, we determined the colony formation ability of the lung cancer cells after knockdown of CEP55. As shown in Fig. 3B, knockdown of CEP55 obviously reduced the size of the single colonies and the number of colonies formed in the A549 and $95 \mathrm{D}$ cells. The number of colonies in the sh- 2 group was apparently smaller than that in the sh-NC group in both cell 
A

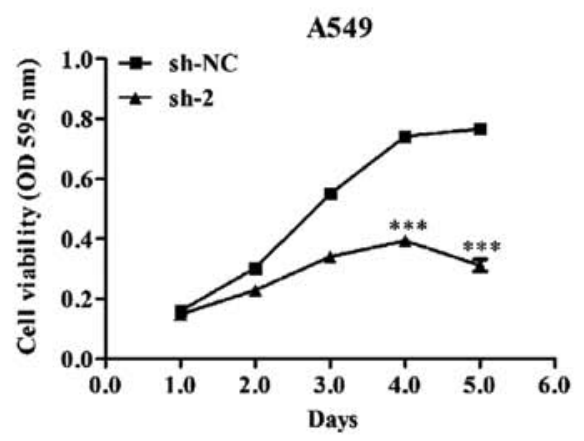

B
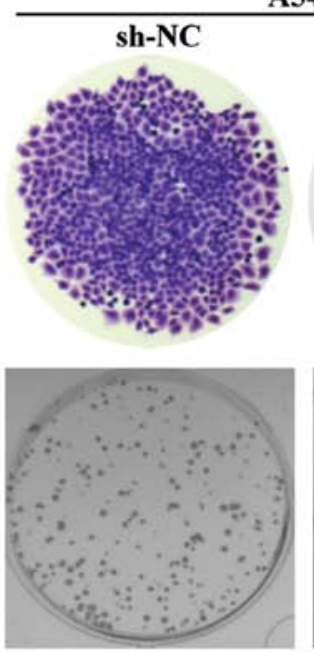

C

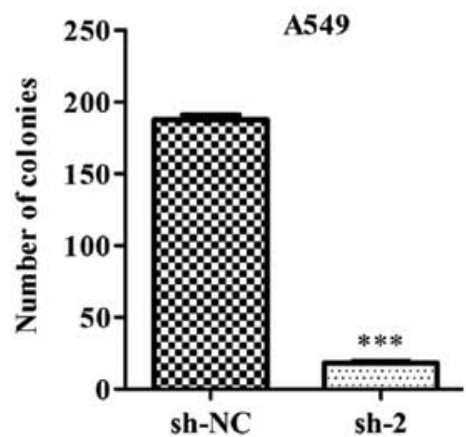

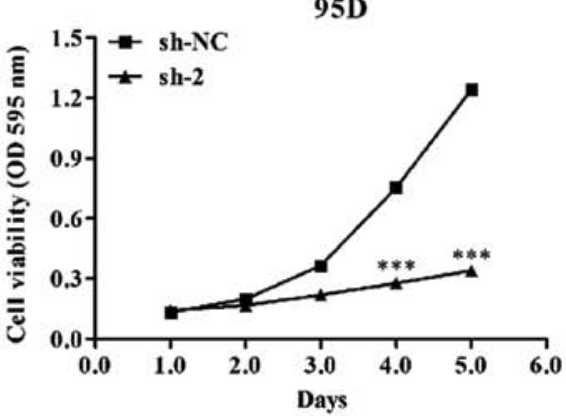

95D
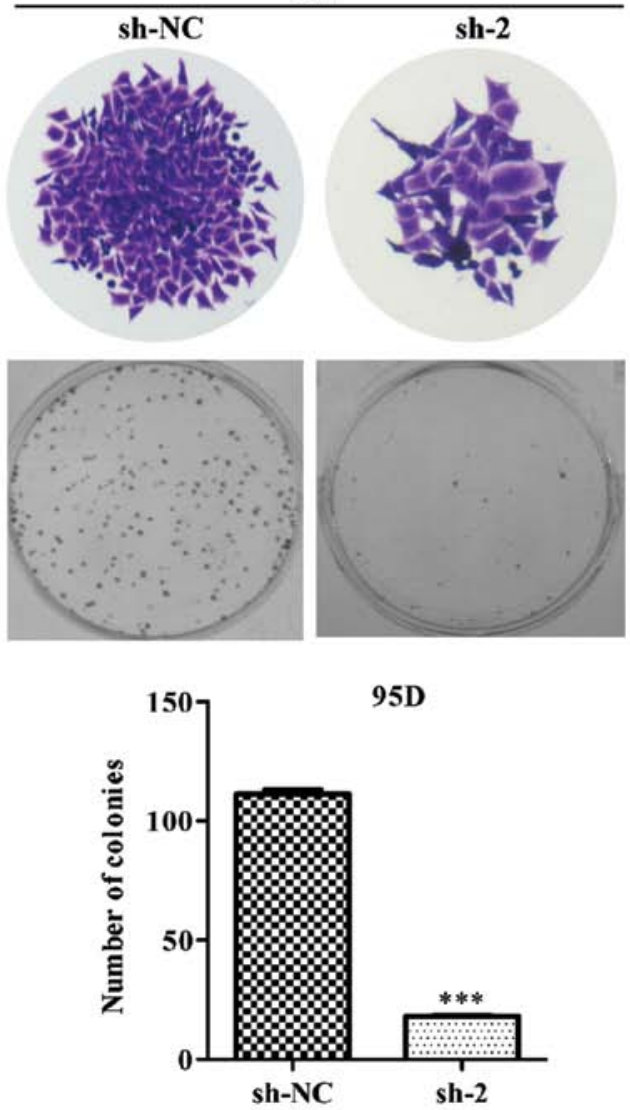

Figure 3. Impaired cell viability and colony formation ability of lung cancer cells after CEP55 knockdown. (A) An MTT assay was used to determine the cell viability in the A549 and 95D cells following transfection with a lentivirus containing sh-2 or sh-NC. (B) Representative images of colonies formed in the A549 and 95D cells with two treatments (sh-2 and sh-NC). (C) Statistical analysis of colony numbers in the A549 and $95 \mathrm{D}$ cells. ${ }^{* * *} \mathrm{P}<0.001$. CEP55, centrosomal protein 55; NC, negative control.

lines (Fig. 3C, p<0.001). These results further indicate that CEP55 acts as a potential tumor gene in lung cancer.

Cell cycle arrest and apoptosis is induced by knockdown of CEP55 in lung cancer cells. To explore the underlying mechanism of reduced cell viability by CEP55 inhibition, flow cytometry was used to analyze the cell cycle distribution in lung cancer cells. The results showed that the percentage of cells in the G1 phase $(\mathrm{p}<0.001)$ was greatly increased in the CEP55-knockdown cells compared to the control cells in both the A549 (Fig. 4A and C) and 95D cell lines (Fig. 4B and D). In addition, more cells were accumulated in the sub-G1 phase in the sh-2 infected 95D cells; much more than those in the
sh-NC infected cells (Fig. 4E, p<0.001). Next, we evaluated cell apoptosis using Annexin V staining and flow cytometry. Our results showed that, the percentages of both early apoptotic (Annexin $\mathrm{V}^{+} / \mathrm{PI}$ ) and late apoptotic (Annexin $\mathrm{V}^{+} / \mathrm{PI}^{+}$) cells in the CEP55 sh-2-transfected cells were increased significantly compared with those in the control shRNA-transfected cells (Fig. 5A). Further analysis indicated that knockdown of CEP55 markedly increased the overall apoptotic cells in the A549 and 95D cell lines (Fig. 5B, p<0.001).

Molecular targets of CEP55 in lung cancer cells. To gain insight into the molecular mechanisms of CEP55 silencing on cell cycle and apoptotic regulation, several molecular 
A

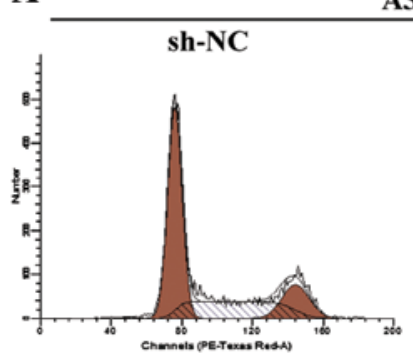

C

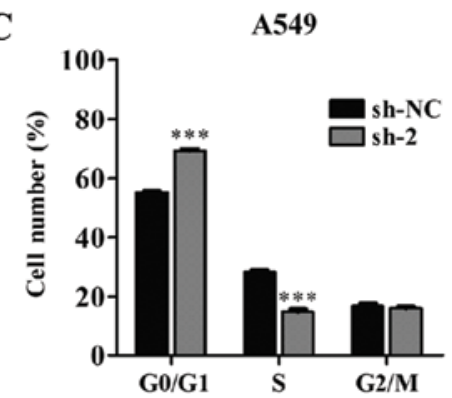

B

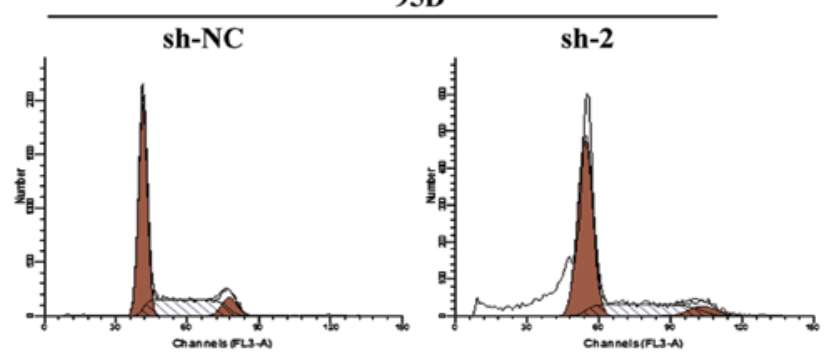

95D

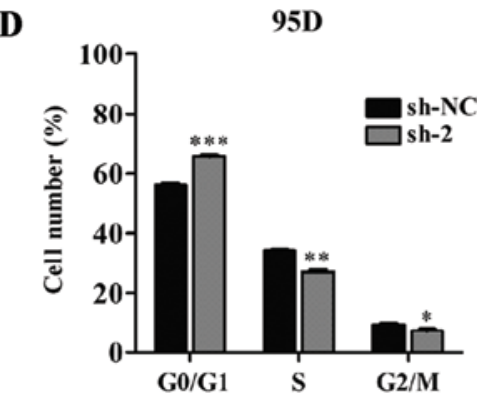

$\mathbf{E}$

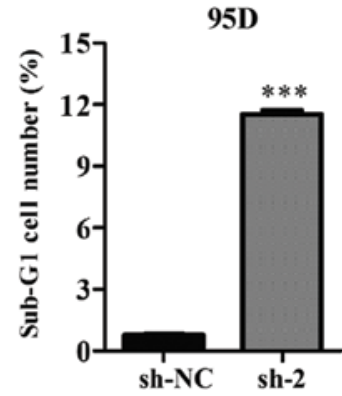

Figure 4. CEP55 knockdown induced G1 phase arrest in lung cancer cells. Flow cytometry was used to analyze cell cycle distribution in the (A) A549 and (B) $95 \mathrm{D}$ cells following transfection with a lentivirus containing sh-2 or sh-NC. Quantitation of cell percentage in each cell cycle phase in (C) A549 and (D) $95 \mathrm{D}$ cells. (E) Percentage of cells in the sub-G1 phase in $95 \mathrm{D}$ cells following transfection with a lentivirus containing sh-2 or sh-NC. $\mathrm{P}<0.05$, ${ }^{* *} \mathrm{p}<0.01$, ${ }^{* * * *} \mathrm{p}<0.001$. CEP55, centrosomal protein 55; NC, negative control.
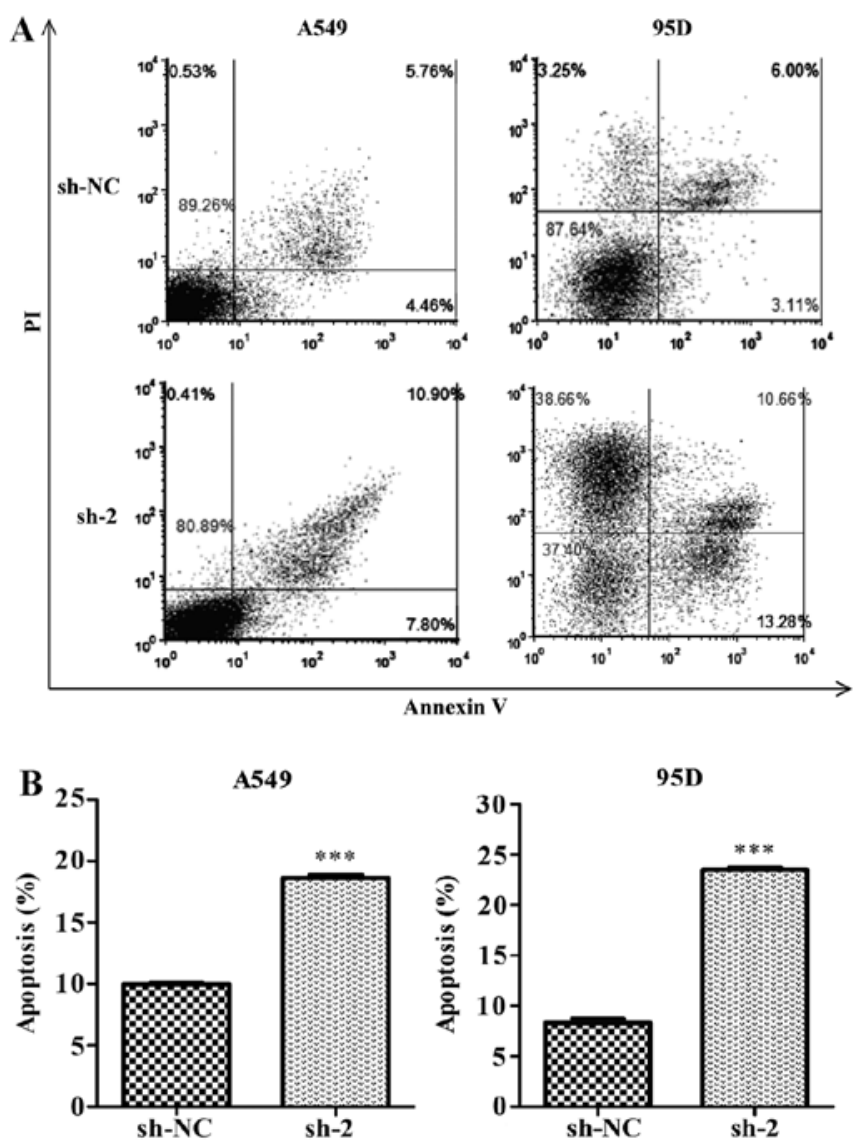

Figure 5. Knockdown of CEP55 induces apoptosis in lung cancer cells. (A) Cell apoptosis was analyzed by Annexin V staining and flow cytometry in the A549 and 95D cells following transfection with a lentivirus containing sh-2 or sh-NC. (B) The overall apoptotic rate, including early and late apoptosis, was assessed in the A549 and 95D cells following transfection with lentivirus containing sh-2 or sh-NC. ${ }^{* * *} \mathrm{P}<0.001$. CEP55, centrosomal protein $55 ; \mathrm{NC}$, negative control.

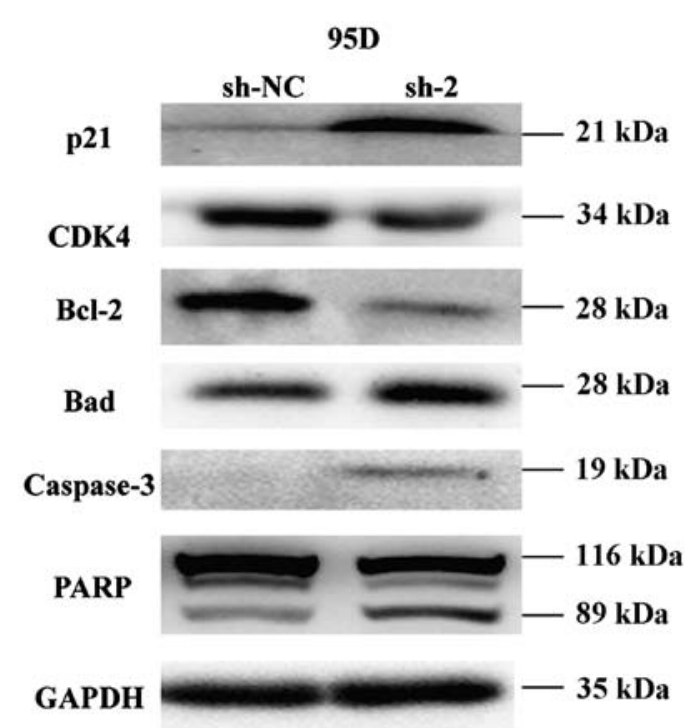

Figure 6. Effect of CEP55 silencing on its downstream molecular targets associated with cell cycle and apoptotic regulation in the 95D cell line, as confirmed by western blot analysis. GAPDH was used as an internal control. CEP55, centrosomal protein 55 .

targets associated with cell cycle and apoptotic regulation were assessed by western blot analysis in the 95D cells stably transfected with sh-2. As shown in Fig. 6, knockdown of CEP55 inhibited expression of CDK4 and Bcl-2 and enhanced expression of p21, cell cycle regulators. Moreover, Bad, caspase-3 and PARP with pro-apoptotic effects were significantly upregulated in the sh-2-transfected cells compared with that in the sh-NC-transfected cells. These results suggest that CEP55 acts as an oncogene by regulating 
the expression of important genes involved in the proliferation and apoptotic pathways.

\section{Discussion}

NSCLC, as a major type, accounts for $>80 \%$ of all lung cancers. Molecular targeted therapy has been widely applied for the treatment of lung cancer with higher efficacy and lower toxicity compared with traditional chemotherapy. Therefore, it is of great importance to identify specific tumor genes and therapeutic targets to improve personalized cancer therapy. CEP55 has been reported to be overexpressed in various human cancer tissues like epithelial ovarian (14) and gastric (9) carcinomas. For these studies, we hypothesized that CEP55 was overexpressed in lung cancer tissues and cell lines. To confirm this hypothesis, we firstly determined the expression of CEP55 in tumor tissues from lung cancer patients and found that it was highly expressed in tumor tissues compared with normal lung tissues. Herein, previous study indicated that VEGFA upregulated the expression of the CEP55 protein, which subsequently resulted in the complex formation with PI3K (11). Therefore, these results suggest a potential oncogenic role of CEP55 in lung cancer, and motivated us to explore its functional significance in lung cancer.

In the present study, the in vitro studies demonstrated that CEP55 knockdown inhibited cell viability and proliferation through the induction of cell cycle arrest and apoptosis in the NSCLC cell lines A549 and 95D, indicating antitumor activity of CEP55 inhibition. Evidence points to the contribution of CEP55 in cell proliferation involved in gastric (9) and breast (10) cancers, as well as nasopharyngeal carcinomas (15). To the best of our knowledge, dysregulation of cell proliferation is the key characteristic of cancer cells, which is closely associated with cell cycle regulation $(16,17)$. As one of the main checkpoints of the cell cycle, the G1/S transition plays a crucial role in the initiation and completion of DNA replication, which is strongly regulated by the $\mathrm{CDK} /$ cyclin complex activity $(18,19)$. In addition CDKs, cyclin-dependent kinase inhibitors (CDKIs) also suppress the G1/S transition in cell cycle regulation (20). Our results showed that cells were arrested in the G1 phase in the CEP55 knocked-down cells. Consistently, further analysis revealed that knockdown of CEP55 downregulated the expression of CDK4 and upregulated the expression of CDKI p21. It has been widely accepted that centrosome-associated proteins play an essential role in cell cycle progression $(21,22)$. Thus, we conclude that CEP55, as a member of the centrosome-associated protein family promotes cell cycle progression in cancer occurrence.

Additionally, we further found that knockdown of CEP55 induced cell apoptosis in the NSCLC cell lines A549 and 95D. Caspase cascade plays a central role in apoptosis, whose activation is regulated by various factors, among which the Bcl-2 family of proteins, as anti-apoptotic mitochondria proteins, are the principle regulators in the intrinsic mitochondrial apoptotic pathway (23). In addition, pro-apoptotic proteins, such as Bad and caspase- 3 may be activated in cell apoptosis $(24,25)$. As the specificity substrate of caspases, PARP plays an important role in DNA repair and can be cleaved by activated caspase-3 resulting in cell apoptosis (26). Recently, it has been reported that CEP55 silencing promoted breast cancer cell apoptosis (10). Consistent with this report, we found that silencing of CEP55 resulted in a significant increase in apoptotic cells in the NSCLC cells. Moreover, the mechanisms involved activation of anti-apoptotic factors, such as Bcl-2, Bad and caspase-3, as well as subsequent amplification of PARP cleavage.

In conclusion, our in vitro data provided enough evidence that the CEP55 level was elevated in lung cancer and its inhibition significantly suppressed lung cancer cell survival and proliferation. These findings suggested that CEP55 may be a potential therapeutic target in lung cancer treatment.

\section{References}

1. Al Zeyadi M, Dimova I, Ranchich V, Rukova B, Nesheva D, Hamude Z, Georgiev S, Petrov D and Toncheva D: Whole genome microarray analysis in non-small cell lung cancer. Biotechnol Biotechnol Equip 29: 111-118, 2015.

2. Ni M, Shi XL, Qu ZG, Jiang H, Chen ZQ and Hu J: Epithelial mesenchymal transition of non-small-cell lung cancer cells A549 induced by SPHK1. Asian Pac J Trop Med 8: 142-146, 2015.

3. Sakai M, Shimokawa T, Kobayashi T, Matsushima S, Yamada Y, Nakamura Y and Furukawa Y: Elevated expression of C10orf3 (chromosome 10 open reading frame 3 ) is involved in the growth of human colon tumor. Oncogene 25: 480-486, 2006.

4. Fabbro M,Zhou BB, Takahashi M, Sarcevic B, Lal P, Graham ME, Gabrielli BG, Robinson PJ, Nigg EA, Ono Y, et al: Cdk1/Erk2- and Plk1-dependent phosphorylation of a centrosome protein, Cep55, is required for its recruitment to midbody and cytokinesis. Dev Cell 9: 477-488, 2005

5. Zhao WM, Seki A and Fang G: Cep55, a microtubule-bundling protein, associates with centralspindlin to control the midbody integrity and cell abscission during cytokinesis. Mol Biol Cell 17: 3881-3896, 2006.

6. Martinez-Garay I, Rustom A, Gerdes HH and Kutsche K: The novel centrosomal associated protein CEP55 is present in the spindle midzone and the midbody. Genomics 87: 243-253, 2006.

7. Singh PK, Srivastava AK, Rath SK, Dalela D, Goel MM and Bhatt ML: Expression and clinical significance of Centrosomal protein 55 (CEP55) in human urinary bladder transitional cell carcinoma. Immunobiology 220: 103-108, 2015.

8. Chen CH, Lu PJ, Chen YC, Fu SL, Wu KJ, Tsou AP, Lee YC, Lin TC, Hsu SL, Lin WJ, et al: FLJ10540-elicited cell transformation is through the activation of PI3-kinase/AKT pathway. Oncogene 26: 4272-4283, 2007.

9. Tao J, Zhi X, Tian Y, Li Z, Zhu Y, Wang W, Xie K, Tang J, Zhang X, Wang L, et al: CEP55 contributes to human gastric carcinoma by regulating cell proliferation. Tumour Biol 35: 4389-4399, 2014

10. Wang Y, Jin T, Dai X and Xu J: Lentivirus-mediated knockdown of CEP55 suppresses cell proliferation of breast cancer cells. Biosci Trends 10: 67-73, 2016.

11. Chen CH, Lai JM, Chou TY, Chen CY, Su LJ, Lee YC, Cheng TS, Hong YR, Chou CK, Whang-Peng J, et al: VEGFA upregulates FLJ10540 and modulates migration and invasion of lung cancer via PI3K/AKT pathway. PLoS One 4: e5052, 2009.

12. Zhang W, Liang Z and Li J: Inhibition of rhotekin exhibits antitumor effects in lung cancer cells. Oncol Rep 35: 2529-2534, 2016.

13. Eskander RN, Randall LM, Sakai T, Guo Y, Hoang B and Zi X: Flavokawain B, a novel, naturally occurring chalcone, exhibits robust apoptotic effects and induces G2/M arrest of a uterine leiomyosarcoma cell line. J Obstet Gynaecol Res 38: 1086-1094, 2012.

14. Zhang W, Niu C, He W, Hou T, Sun X, Xu L and Zhang Y: Upregulation of centrosomal protein 55 is associated with unfavorable prognosis and tumor invasion in epithelial ovarian carcinoma. Tumour Biol 37: 6239-6254, 2016.

15. Chen CH, Shiu LY, Su LJ, Huang CY, Huang SC, Huang CC, Yin YF, Wang WS, Tsai HT, Fang FM, et al: FLJ10540 is associated with tumor progression in nasopharyngeal carcinomas and contributes to nasopharyngeal cell proliferation, and metastasis via osteopontin/CD44 pathway. J Transl Med 10: 93, 2012. 
16. Nguyen-Ba G and Vasseur P: Epigenetic events during the process of cell transformation induced by carcinogens (Review). Oncol Rep 6: 925-932, 1999.

17. Vermeulen K, Van Bockstaele DR and Berneman ZN: The cell cycle: A review of regulation, deregulation and therapeutic targets in cancer. Cell Prolif 36: 131-149, 2003.

18. Massagué J: G1 cell-cycle control and cancer. Nature 432: 298-306, 2004

19. Cicenas J, Kalyan K, Sorokinas A, Jatulyte A, Valiunas D, Kaupinis A and Valius M: Highlights of the latest advances in research on CDK Inhibitors. Cancers (Basel) 6: 2224-2242, 2014

20. Lim S and Kaldis P: Cdks, cyclins and CKIs: Roles beyond cell cycle regulation. Development 140: 3079-3093, 2013.

21. Delattre M and Gönczy P: The arithmetic of centrosome biogenesis. J Cell Sci 117: 1619-1630, 2004.
22. Srsen V and Merdes A: The centrosome and cell proliferation. Cell Div 1: 26, 2006.

23. Dejean LM, Martinez-Caballero S, Manon S and Kinnally KW: Regulation of the mitochondrial apoptosis-induced channel, MAC, by BCL-2 family proteins. Biochim Biophys Acta 1762: 191-201, 2006.

24. Manning BD and Cantley LC: AKT/PKB signaling: Navigating downstream. Cell 129: 1261-1274, 2007.

25. Zhang X, Tang N, Hadden TJ and Rishi AK: Akt, FoxO and regulation of apoptosis. Biochim Biophys Acta 1813: 1978-1986, 2011.

26. Yu SW, Andrabi SA, Wang H, Kim NS, Poirier GG, Dawson TM and Daws on VL: Apoptosis-inducing factor mediates poly(ADP-ribose) (PAR) polymer-induced cell death. Proc Natl Acad Sci USA 103: 18314-18319, 2006. 